\title{
Experimental Observation of Spatially Localized Dynamo Magnetic Fields
}

\author{
B. Gallet, ${ }^{1}$ S. Aumaître, ${ }^{2}$ J. Boisson, ${ }^{2}$ F. Daviaud, ${ }^{2}$ B. Dubrulle, ${ }^{2}$ N. Bonnefoy, ${ }^{3}$ M. Bourgoin,,${ }^{4}$ Ph. Odier,,${ }^{5} .-F$. Pinton, ${ }^{5}$ \\ N. Plihon, ${ }^{5}$ G. Verhille, ${ }^{6}$ S. Fauve, ${ }^{1}$ and F. Pétrélis ${ }^{1}$ \\ ${ }^{1}$ Laboratoire de Physique Statistique, Ecole Normale Supérieure, CNRS, Université Pierre et Marie Curie, \\ Université Paris Diderot, Paris, France \\ ${ }^{2}$ Service de Physique de l'État Condensé, CNRS \& CEA Saclay, F-91191 Gif-sur-Yvette, France \\ ${ }^{3}$ CEA/CE Cadarache, DTN/STPA/LIET, 13108 St. Paul lez Durance Cedex, France \\ ${ }^{4}$ Laboratoire des Ecoulements Géophysiques et Industriels, CNRS \& Université Joseph Fourier, \\ BP 53, F-38041 Grenoble cedex 9, France \\ ${ }^{5}$ Laboratoire de Physique de l'École Normale Supérieure de Lyon, CNRS \& Université de Lyon, 46 allée d'Italie, \\ F-69364 Lyon cedex 07, France \\ ${ }^{6}$ Institut de Recherche sur les Phénomènes Hors Equilibre, CNRS \& Université d'Aix-Marseille, \\ 49 rue Frédéric Joliot Curie, B. P. 146, 13384 Marseille cedex 13, France
}

(Received 19 January 2012; published 2 April 2012)

\begin{abstract}
We report the first experimental observation of a spatially localized dynamo magnetic field, a common feature of astrophysical dynamos and convective dynamo simulations. When the two propellers of the von Kármán sodium experiment are driven at frequencies that differ by $15 \%$, the mean magnetic field's energy measured close to the slower disk is nearly 10 times larger than the one close to the faster one. This strong localization of the magnetic field when a symmetry of the forcing is broken is in good agreement with a prediction based on the interaction between a dipolar and a quadrupolar magnetic mode.
\end{abstract}

DOI: 10.1103/PhysRevLett.108.144501

PACS numbers: $47.65 .-\mathrm{d}$

The magnetic fields of many planets and stars are generated by the motion of an electrically conducting fluid through a dynamo process [1]. Hemispherical localization of the magnetic field is a commonly observed feature. At the end of Maunder's minimum, there were very few sunspots and these were localized only in the southern hemisphere of the Sun [2]. This localization of the active regions corresponds to a north-south asymmetry of the large-scale solar magnetic field. Another astrophysical example is the remanent magnetic field of Mars, which is also localized in the southern hemisphere [3]. Although Mars is not a dynamo anymore, it has been suggested that it used to be, with a dynamo magnetic field much stronger in the southern hemisphere of the planet [4]. As far as numerical dynamo models are concerned, the first observation of hemispherical magnetic fields was reported by Grote and Busse [5] in a simulation of convective dynamo between two spheres. Such a localization has been observed more recently by Landeau and Aubert using different thermal boundary conditions and removing the inner sphere [6]. Analytical calculations using simple $\alpha^{2}$ models showed that hemispherical localization of the magnetic field generically results from a weak north-south symmetry breaking of the flow [7]. When a dipolar and a quadrupolar magnetic mode are both close to onset, a tiny symmetry breaking couples the two modes and leads to a strong hemispherical localization of the magnetic field: such a localized field has much less magnetic energy in one hemisphere than in the other, the ratio between the two depending on the precise structures of the underlying dipolar and quadrupolar modes. Depending on the way the symmetry is broken, this localization may or may not be followed by magnetic reversals at higher amplitude of the symmetrybreaking perturbation. The hemispherical localization of the magnetic field observed in astrophysical objects as well as in DNSs could thus be the consequence of a slight equatorial symmetry breaking of the flow. Here we report the experimental observation of a spatially localized dynamo magnetic field generated in a swirling flow of liquid sodium when a symmetry of the forcing is broken.

The von Kármán sodium (VKS) experiment consists of two coaxial impellers counterrotating in a cylindrical tank filled with liquid sodium (inset of Fig. 1). The operating temperature is $120^{\circ} \mathrm{C}$, which corresponds to an electrical conductivity of $9.6 \times 10^{6} \mathrm{~S} \cdot \mathrm{m}^{-1}$ for the sodium. The cylindrical vessel has radius $289 \mathrm{~mm}$ and length $604 \mathrm{~mm}$. The impellers are made of soft iron disks (radius $154.5 \mathrm{~mm}$ ) fitted with eight curved blades. They are $371 \mathrm{~mm}$ apart. The magnetic measurements are performed using two arrays of ten three-axis Hall probes, radially aligned close to each one of the two disks (109 mm away from the midplane, deepest probe $63 \mathrm{~mm}$ away from the cylinder's axis then one probe every $28 \mathrm{~mm}$ ). In the following we use Cartesian coordinates $(x, y, z)$, with $x$ along the axis of the cylinder. When the two propellers counterrotate at the same frequency $F_{1}=F_{2}$, the setup is invariant to a rotation of angle $\pi$ around a radial axis in the equatorial plane of the cylinder (denoted as $\mathcal{R}_{\pi}$ ). Above a critical rotation frequency $F_{1}=F_{2} \simeq 12 \mathrm{~Hz}$, this configuration produces a fluctuating dynamo magnetic field which large-scale structure is a dipole aligned with the axis of the cylinder [8]. The mean magnetic field is axisymmetric 


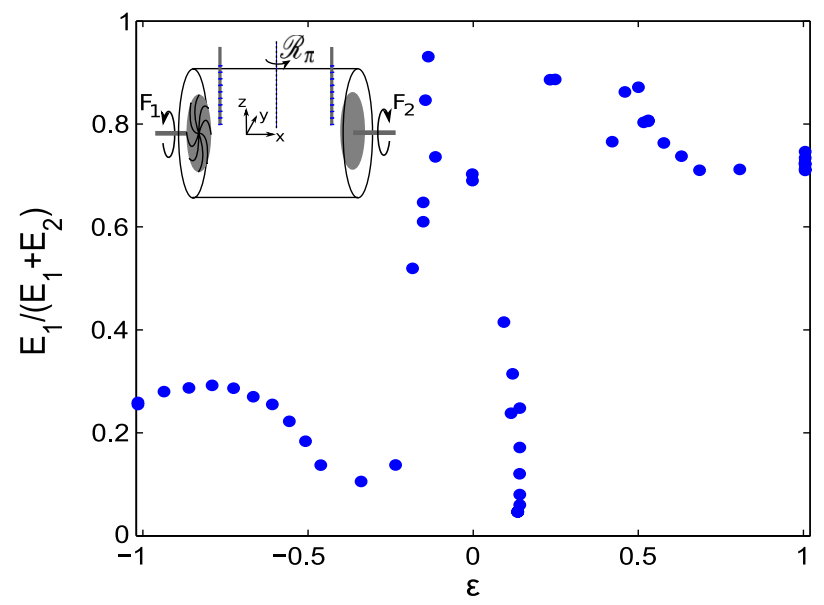

FIG. 1 (color online). Energy fraction close to disk 1 as a function of the dimensionless symmetry-breaking amplitude. All stationary dynamos obtained for $\max \left(F_{1}, F_{2}\right) \geq 22.75 \mathrm{~Hz}$ are shown. Inset: sketch of the experiment: two impellers counterrotate at frequencies $F_{1}$ and $F_{2}$. When $F_{1}=F_{2}$ the setup is invariant to $\mathcal{R}_{\pi}$ rotation. The vertical thick lines indicate the locations of the two arrays of three-axis Hall probes which measure the magnetic field close to each disk.

to a good approximation, and despite the turbulent fluctuations the axial dipole remains of constant polarity. By contrast, when the propellers are driven at different frequencies $F_{1} \neq F_{2}$, the $\mathcal{R}_{\pi}$ symmetry is broken and regimes of periodic or erratic field reversals can be observed [9].

The VKS setup is thus well suited to study the influence of symmetry breaking on the large-scale geometry of the magnetic field. The frequency detuning $\epsilon=\left(F_{1}-\right.$ $\left.F_{2}\right) /\left(F_{1}+F_{2}\right)$ is used to quantify the asymmetry of the forcing, $\epsilon=0$ corresponding to exact counterrotation and $\epsilon= \pm 1$ to the situation where only one disk rotates. We study the response of the dynamo field to this forcing asymmetry by looking at the asymmetry of the magnetic field measured by the two arrays of probes. Note that the hydrodynamic response to this forcing asymmetry is large as far as the location of the shear layer is concerned. It moves toward the slower disk and reaches it for $|\epsilon| \simeq 0.1$ in a similar geometry where two different flow structures have been observed: two counterrotating toroidal eddies in the vicinity of exact counterrotation or a single eddy rotating with the faster disk for larger values of $|\epsilon|$ [10].

The magnetic field generated by the flow is averaged in time, squared, and summed over the probes of each array. The result is denoted as $E_{1}$ (respectively $E_{2}$ ). The subscript refers to the disk close to which the measurement is performed. In order to quantify the localization of the magnetic energy close to one disk, or its asymmetry with respect to $\mathcal{R}_{\pi}$, we compute the ratio $E_{1} /\left(E_{1}+E_{2}\right)$, which is displayed in Fig. 1. We observe that when the two disks counterrotate at the same velocity, $E_{1} \sim E_{2}$. Then, $E_{1} /\left(E_{1}+E_{2}\right)$ deviates from 0.5 when $|\epsilon|$ is increased,

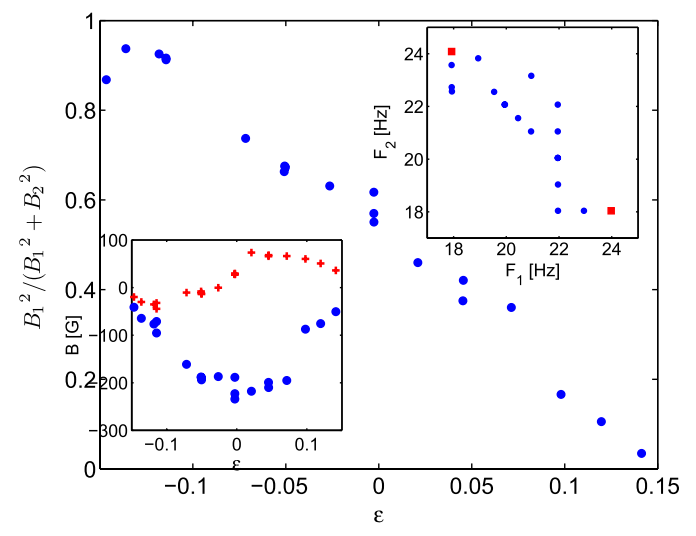

FIG. 2 (color online). Asymmetry factor of the magnetic field as a function of the dimensionless symmetry-breaking amplitude. At $|\epsilon| \simeq 15 \%$, the magnetic energy close to the slower disk is roughly 10 times larger than the one close to the faster disk. Inset (top): parameter space of the measurement points used for this graph (O: steady magnetic field; $\mathbf{\square}$ : randomly reversing magnetic field); (bottom): amplitudes of the dipolar and quadrupolar components evaluated by the sum and difference of axial fields measured by the deepest probe of each array $(0: d ;+q)$.

the magnetic field becoming larger close to the slower disk. When $|\epsilon|$ is increased further the ratio $E_{1} /\left(E_{1}+E_{2}\right)$ displays an abrupt jump in the range $0.15<|\epsilon|<0.2$, the magnetic field becoming localized close to the faster disk. Finally, when only one disk rotates $(\epsilon= \pm 1)$, the dynamo field is larger in the vicinity of that disk. Note that the asymmetry of the field is stronger for $|\epsilon| \sim 0.1$ than when only one disk rotates.

The asymmetry of the magnetic field as $|\epsilon|$ is increased from zero could be due to a modification of the induction processes that results from the displacement of the shear layer, which moves toward the slower disk. Despite this change in the mean flow geometry, the relative difference in the torques $C_{i}$ applied by the two disks remains small, $C_{1} /\left(C_{1}+C_{2}\right) \sim 0.5+0.7 \epsilon$ for $|\epsilon|$ smaller that 0.1 . $C_{1} /\left(C_{1}+C_{2}\right)$ slightly increases in slope just below $|\epsilon|=$ 0.1 and then displays an inflection point around $|\epsilon|=0.2$, which can be both related to modifications of the flow structure. The detailed structure of the flow as $|\epsilon|$ is increased being not known, we cannot determine how the abrupt jump displayed by $E_{1} /\left(E_{1}+E_{2}\right)$ is related to the modification of the induction processes.

We focus hereafter on measurements performed in the vicinity of exact counterrotation $\left(\left|F_{1}-F_{2}\right| \ll\left|F_{1}+F_{2}\right|\right)$, the values of $F_{1}$ and $F_{2}$ being close to $21 \mathrm{~Hz}$ and spanning the small domain of symmetry breaking beyond which random reversals are observed (top inset of Fig. 2). Instead of the magnetic energy averaged on the array of probes, we consider the square of the time-averaged magnetic field of the deepest probe of each array, $B_{1,2}^{2}=$ $\left\|\overline{\mathbf{B}}_{1,2}\right\|^{2}$. The ratio $B_{1}^{2} /\left(B_{1}^{2}+B_{2}^{2}\right)$, hereafter named the asymmetry factor, is displayed in Fig. 2 as a function of $\epsilon$. The time-averaged magnetic field having mostly a 
large-scale structure, the asymmetry characterization using only the deepest probes gives a behavior similar to the ones based on the magnetic energy averaged over the whole probe arrays. However, the magnetic field measured on the deepest probes is better suited for extracting the dipolar and quadrupolar components (see below). In exact counterrotation the asymmetry factor is approximately 0.5 , which corresponds to the $\mathcal{R}_{\pi}$-antisymmetric axial dipole. As the symmetry-breaking amplitude increases, the magnetic energy gets strongly localized close to one of the two disks: a significant localization is already observed for $\epsilon=0.05$. For $\epsilon=0.15$, the magnetic energy density measured close to the slower disk is roughly 10 times larger than the one measured close to the faster disk. The two different ways to define the asymmetry of the magnetic field used in Figs. 1 and 2 display the same qualitative behavior as $\epsilon$ is varied. Note that they both give a value of the asymmetry parameter larger than 0.5 for $\epsilon=0$. This presumably results from small asymmetries in the experimental setup and in the location of the probes.

We now wish to stress the fact that two magnetic modes are required to describe these observations. If only one (dipolar) magnetic mode were close to onset, a symmetry breaking of dimensionless amplitude $\epsilon \ll 1$ would lead perturbatively to a modification of order $\epsilon$ in the structure of the eigenmode; hence, we certainly would not expect a stronger localization for $\epsilon=0.15$ than in the case of only one rotating disk.

Let us assume that two magnetic modes are close to onset in the counterrotating regime. One is of dipolar symmetry, and grows in exact counterrotation above a critical frequency $F_{1}=F_{2}=F^{(d)}$. The second one is of quadrupolar symmetry, and gets linearly unstable above a critical frequency $F^{(q)}$, with $F^{(q)}>F^{(d)}$ and $F^{(q)}-F^{(d)} \ll F^{(d)}$. There is thus a new dimensionless parameter $\left(F^{(q)}-F^{(d)}\right) / F^{(d)}$ to which $\epsilon$ should be compared. This new parameter is small because the two modes have close dynamo thresholds. Let us write the large-scale magnetic field $\mathbf{B}(\mathbf{x}, t)=d(t) \mathbf{D}(\mathbf{x})+q(t) \mathbf{Q}(\mathbf{x})$ and define $A(t)=d(t)+i q(t)$. At linear order, the equation for this complex amplitude reads

$$
\dot{A}=\left(\mu_{r}+i \mu_{i}\right) A+\left(\nu_{r}+i \nu_{i}\right) \bar{A},
$$

where the coefficients $\mu_{r}, \mu_{i}, \nu_{r}$, and $\nu_{i}$ are real. In exact counterrotation the $\mathcal{R}_{\pi}$ symmetry imposes $\mu_{i}=\nu_{i}=0$, and the dipolar and quadrupolar modes have, respectively, the growth rates $\mu_{r}+\nu_{r}$ and $\mu_{r}-\nu_{r}$. The coefficient $\nu_{r}$ is thus proportional to the difference in onsets of the dipolar and quadrupolar modes. When $\mathcal{R}_{\pi}$ is weakly broken the coefficients $\mu_{i}$ and $\nu_{i}$ are proportional to the symmetrybreaking amplitude $\epsilon$. The phase $\theta$ of the complex amplitude $A$ follows the equation

$$
\dot{\theta}=\mu_{i}+\nu_{i} \cos (2 \theta)-\nu_{r} \sin (2 \theta) .
$$

When $\epsilon=0$, a pure dipolar mode $(\theta=0)$ is stable (with respect to the quadrupole) provided $\nu_{r}>0$. The eigenmode becomes a mix of dipolar and quadrupolar field as $\epsilon$ increases, until $\mu_{i}^{2}=\nu_{r}^{2}+\nu_{i}^{2}$ where a saddle-node bifurcation occurs: the solution of the phase equation becomes time dependent and the magnetic field reverses periodically in time. This obviously requires that $\mu_{i}$ increases faster than $\nu_{i}$ when $\epsilon$ increases. Just before the saddle-node bifurcation, turbulent fluctuations of the flow can trigger random reversals of the magnetic field [11]. If, for simplicity, we assume that $\mu_{i}$ increases much faster than $\nu_{i}$, the (stationary) phase equation reads at the onset of the saddle-node bifurcation $\pm \nu_{r}-$ $\nu_{r} \sin (2 \theta) \simeq 0$, which solution is $\theta=\pi / 4[\pi]$ or $\theta=$ $-\pi / 4[\pi]$, depending on the relative signs of $\mu_{i}$ and $\nu_{r}$. These solutions correspond to $d= \pm q$ : the dipolar and quadrupolar components have equal or opposite amplitudes, and the corresponding eigenmode is localized in a half cylinder close to one of the two disks [7]. As a consequence, in a reversing regime the magnetic field is spatially localized during the long phases of constant polarity. Notice that partial localization occurs when $\mu_{i}$ or $\nu_{i}$ is of the order of $\nu_{r}$. In terms of the dimensionless parameters written above, this corresponds to $\left(F_{1}-F_{2}\right) /\left(F_{1}+F_{2}\right) \sim\left(F^{(q)}-F^{(d)}\right) / F^{(d)} \ll 1$ : if the onsets of the dipolar and quadrupolar modes are close enough, a tiny symmetry breaking strongly localizes the magnetic field close to one of the two disks.

The bottom inset in Fig. 2 shows the amplitudes $d$ and $q$ of the dipolar and quadrupolar components evaluated, respectively, as the sum $\bar{B}_{x}^{(1)}+\bar{B}_{x}^{(2)}$ and the difference $\bar{B}_{x}^{(1)}-\bar{B}_{x}^{(2)}$ of the time-averaged axial fields measured by the deepest probe of each array. The resulting $d$ and $q$ are multiplied by the sign of $d$ to collapse the $+\mathbf{B}$ and -B dynamo branches. This highlights the opposite parities of $d$ and $q$ with respect to $\epsilon$. Localization occurs together with $d \simeq q$ around $\epsilon=-0.15$ and $d \simeq-q$ around $\epsilon=+0.15$.

When the asymmetry of the forcing is increased further, field reversals are observed. In the framework of the phase equation (2), we are thus in the situation where $\mu_{i}$ increases faster than $\nu_{i}$. A time series of such a reversing regime is displayed in Fig. 3 for $F_{1}=18 \mathrm{~Hz}$ and $F_{2}=$ $24 \mathrm{~Hz}$. We plot the axial field close to disk $1, B_{1}^{2}$ and $B_{2}^{2}$, and the ratio $B_{2}^{2} / B_{1}^{2}$. The system spends a long time in a given polarity, with the magnetic energy localized close to the slower disk $\left(B_{1}^{2} \gg B_{2}^{2}\right)$. During a reversal, it gets briefly localized close to the faster disk (large value of the ratio $B_{2}^{2} / B_{1}^{2}$ ) before coming back close to the slower disk, with the opposite polarity $\left(B_{1}^{2} \gg B_{2}^{2}\right.$, but $B_{x}^{(1)}$ has changed sign). This can be understood from the dipolequadrupole model since these two components do not change sign simultaneously during a reversal as displayed in Fig. 4 . In the $(d, q)$ plane, a reversal is related to the path from the point $M$ to $-M$. These points are in 

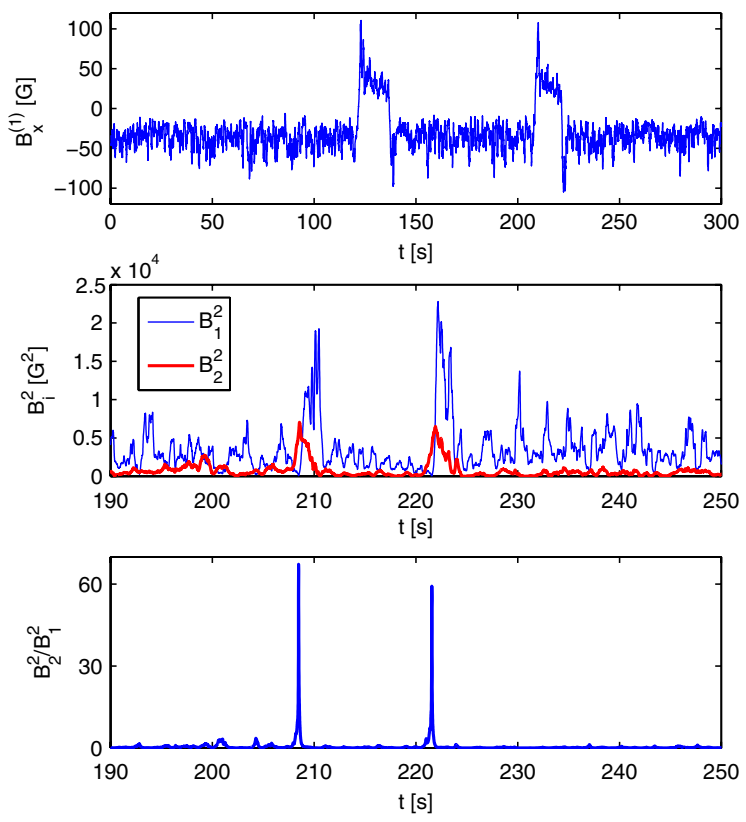

FIG. 3 (color online). Time series in reversing regime at $F_{1}=18 \mathrm{~Hz}$ and $F_{2}=24 \mathrm{~Hz}$ (Top: axial field close to disk 1; middle: magnetic energy density for a zoom on the last two reversals; bottom: ratio of these two energies.) Before computing the energies, we use a sliding average on $500 \mathrm{~ms}$ to remove high frequency fluctuations. The magnetic field is localized close to the slower disk during the phases of given polarity. It gets briefly localized close to the faster disk during a reversal.

the vicinity of the $d=q$ line for which the magnetic field is larger close to the slower disk. The path from $M$ to $-M$ crosses the $d=-q$ line for which only one component (the dipole or the quadrupole) is reversed. The magnetic field is then larger close to the faster disk. As also predicted from (2), at the onset of reversals the field is localized during the long phases of given polarity. We also note that there is a small asymmetry between one polarity and the other. It may be an effect of Earth's magnetic field breaking the $\mathbf{B} \rightarrow-\mathbf{B}$ symmetry, so that the onset of saddle-node bifurcation is slightly smaller for $B_{x}^{(1)}>0$ than for $B_{x}^{(1)}<0$.

Finally, we can understand the abrupt change in the asymmetry parameter when $|\epsilon|$ is increased above 0.15 using a similar argument. This transition is observed when $|\epsilon|$ is increased above the regime that involves field reversals. The system undergoes a transition to a stationary dynamo that differs from the one observed in the vicinity of exact counterrotation. Its fixed points are located in different quadrants of the $(d, q)$ plane of Fig. 4 ( $X$ signs instead of $\pm M$ ). Thus the localization of the magnetic field changes from the slower to the faster disk. This scenario can be easily described in the framework of Eq. (2) if $\nu_{r}$ decreases and changes sign when $|\epsilon|$ increases. A saddlenode bifurcation from stationary to reversing solutions occurs for $\mu_{i}^{2}=\nu_{r}^{2}+\nu_{i}^{2}$. As $\epsilon$ is increased further, a

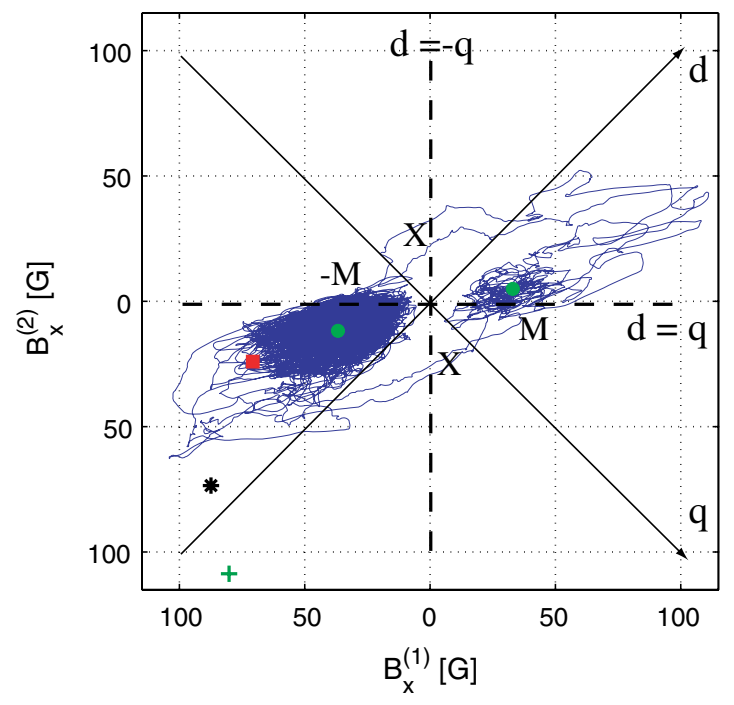

FIG. 4 (color online). Evolution in phase space $\left(B_{x}^{(1)}, B_{x}^{(2)}\right)$ as the symmetry-breaking amplitude increases. The symbols are the time averages $\bar{B}_{x}^{(1)}$ and $\bar{B}_{x}^{(2)}$ for $+:\left(F_{1}, F_{2}\right)=(21 \mathrm{~Hz}, 21 \mathrm{~Hz}) ; *$ : $\left(F_{1}, F_{2}\right)=(19.5 \mathrm{~Hz}, 22.5 \mathrm{~Hz}) ; \mathbf{\square}:\left(F_{1}, F_{2}\right)=(19 \mathrm{~Hz}, 24 \mathrm{~Hz})$. The line is a trajectory during reversals at $\left(F_{1}, F_{2}\right)=$ $(18 \mathrm{~Hz}, 24 \mathrm{~Hz})$, and the circles are the corresponding averages computed for each polarity $( \pm M)$.

transition back to stationary solutions occurs and the two sets of stationary solutions are located in different quadrants of the $(d, q)$ plane [12].

We have reported the first experimental observation of a spatially localized magnetic field. The strong localization of the VKS magnetic field when the $\mathcal{R}_{\pi}$ symmetry is broken can be interpreted as the competition between a dipolar and a quadrupolar magnetic mode which dynamo thresholds are close. This mechanism is summarized in the phase space $\left(B_{x}^{(1)}, B_{x}^{(2)}\right)$ shown in Fig. 4: in exact counterrotation the field is an $\mathcal{R}_{\pi}$-antisymmetric axial dipole, which representative point is on the bisector $B_{x}^{(1)}=B_{x}^{(2)}$ (up to slight imperfections in the experimental setup, and Earth's magnetic field). As we move away from counterrotation, this dipolar field gets coupled to a quadrupolar mode, and the representative point moves away from the bisector, towards $B_{x}^{(2)}=0$ : the magnetic field is localized close to the slower disk. As predicted from the amplitude equation, at the onset of the reversing regime the system spends long periods of time in this localized state, before reversing and getting localized on the same side, but with the other polarity.

We thank M. Moulin, C. Gasquet, A. Skiara, D. Courtiade, J.-F. Point, P. Metz, V. Padilla, and M. Tanase for their technical assistance and the Dynamo GDRE. This work is supported by ANR 08-0039-02, Direction des Sciences de la Matière and Direction de l'Energie Nucléaire of CEA, Ministère de la Recherche and CNRS. The experiment is operated at CEA/Cadarache DEN/DTN. 
[1] Ya. B. Zeldovich, A. A. Ruzmaikin, and D. D. Sokoloff, Magnetic Fields In Astrophysics (Gordon and Breach, New York, 1983).

[2] J. C. Ribes and E. Nesme-Ribes, Astron. Astrophys. 276, 549 (1993); D. Sokoloff and E. Nesme-Ribes, Astron. Astrophys. 288, 293 (1994).

[3] B. Langlais, M. E. Purucker, and M. Mandea, J. Geophys. Res. Planets 109, E02008 (2004); B. Langlais and H. Amit, Science 321, 1784 (2008).

[4] S. Stanley et al., Science 321, 1822 (2008).

[5] E. Grote and F. H. Busse, Phys. Rev. E 62, 4457 (2000).
[6] M. Landeau and J. Aubert, Phys. Earth Planet. Inter. 185, 61 (2011).

[7] B. Gallet and F. Pétrélis, Phys. Rev. E 80, 035302(R) (2009).

[8] F. Ravelet et al., Phys. Rev. Lett. 101, 074502 (2008).

[9] M. Berhanu et al., Europhys. Lett. 77, 59001 (2007).

[10] P.-P. Cortet et al., Phys. Fluids 21, 025104 (2009).

[11] F. Pétrélis and S. Fauve, J. Phys. Condens. Matter 20, 494203 (2008); F. Pétrélis, S. Fauve, E. Dormy, and J.-P. Valet, Phys. Rev. Lett. 102, 144503 (2009).

[12] F. Pétrélis and S. Fauve, arXiv:0806.3937. 